\title{
FUNDAMENTALS OF
} INTERFEROMETRIC GRAVITATIONAL WAVE DETECTORS 
This page is intentionally left blank 


\section{FUNDAMENTALS OF}

\section{Peter R. Saulson}

Department of Physics

Syracuse University, USA 


\section{Published by}

World Scientific Publishing Co. Pte. Ltd.

P O Box 128, Farrer Road, Singapore 9128

USA office: Suite 1B, 1060 Main Street, River Edge, NJ 07661

घ $\mathrm{G} K$ office: 73 Lynton Mead, Totteridge, London N20 8DH

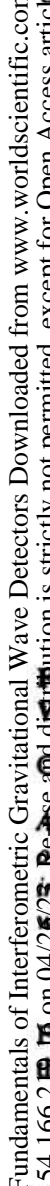

ISBN: 981-02-1820-6

Printed in Singapore. 


\section{Contents}

\section{Preface}

1 The Search for Gravitational Waves 1

1.1 The Importance of the Search . . . . . . . . . . . . . . 1

1.2 A Bit of History . . . . . . . . . . . . . . . 2

1.3 The Practice of Gravitational Wave Detection . . . . . . . . . . 4

1.4 A Guide for the Reader . . . . . . . . . . . . . . . . . 6

2 The Nature of Gravitational Waves 9

2.1 Waves in General Relativity . . . . . . . . . . . . . . . 9 9

2.2 The Michelson-Morley Experiment . . . . . . . . . . . . . . 12

2.3 A Schematic Detector of Gravitational Waves . . . . . . . . . . . 18

2.4 Description of Gravitational Waves in Terms of Force . . . . . . . 25

3 Sources of Gravitational Waves 29

3.1 Physics of Gravitational Wave Generation . . . . . . . . . . 29

3.2 In the Footsteps of Heinrich Hertz? . . . . . . . . . . . . . . 33

3.3 Observation of Gravitational Wave Emission . . . . . . . . . . 36

3.4 Astronomical Sources of Gravitational Waves . . . . . . . . . . . 38

3.4 .1 Neutron star binaries . . . . . . . . . . . . . . . 39

3.4 .2 Supernovae . . . . . . . . . . . . . 40

3.4 .3 Pulsars. . . . . . . . . . . . . . . . . . . 42

3.4.4 "Wagoner stars" $\ldots \ldots \ldots \ldots \ldots \ldots$

3.4.5 Black holes ............ . . . . . . . 44

3.4.6 Stochastic backgrounds . . . . . . . . . . . . . 46

3.4 .7 Discussion . . . . . . . . . . . . . . . . . 46

4 Linear Systems, Signals and Noise 49

4.1 Characterizing a Time Series . . . . . . . . . . . . . . . . . . . 49

4.1 .1 The Fourier transform . . . . . . . . . . . . . . 50

4.1.2 Cross-correlation and autocorrelation . . . . . . . . 50

4.1 .3 Convolution . . . . . . . . . . . . . . . 52

4.1.4 The power spectrum $\ldots \ldots \ldots \ldots \ldots \ldots \ldots \ldots \ldots$ 
4.1.5 The Periodogram ... . . . . . . . . . . . . 53

4.1.6 Interpretation of power spectra . . . . . . . . . 53

4.1.7 The amplitude spectral density . . . . . . . . . . . 55

4.2 Linear Systems . . . . . . . . . . . . . . . . 55

4.2 .1 Bode plots . . . . . . . . . . . . . . . 61

4.2 .2 Frequency response example . . . . . . . . . . . . 61

4.3 The Signal-to-Noise Ratio . . . . . . . . . . . . . . . 62

4.3 .1 Noise statistics . . . . . . . . . . . . . . . . 62

4.3.2 Matched templates and matched filters . . . . . . . . . . 66

4.3.3 SNR rules of thumb. . . . . . . . . . . . . . . . . 68

4.3.4 The characteristic amplitude . . . . . . . . . . 69

$\begin{array}{ll}\text { Optical Readout Noise } & 71\end{array}$

5.1 Photon Shot Noise . . . . . . . . . . . . . . . 71

5.2 Radiation Pressure Noise . . . . . . . . . . . . . . . 75

5.3 Shot Noise in Classical and Quantum Mechanics . . . . . . . . . 80

5.4 The Remarkable Precision of Interferometry . . . . . . . . . . 83

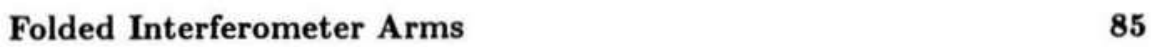

6.1 Herriott Delay Line . . . . . . . . . . . . . . . . . 86

6.2 Beam Diameter and Mirror Diameter . . . . . . . . . . 88

6.3 Fabry-Perot Cavities . . . . . . . . . . . . . . . . 90

6.4 A Long Fabry-Perot Cavity . . . . . . . . . . . . . . . . . 97

6.5 Hermite-Gaussian Beams . . . . . . . . . . . . . . . 97

6.6 Scattered Light in Interferometers . . . . . . . . . . . . . 99

6.7 Comparison of Fabry-Perot Cavities with Delay Lines . . . . . . . . 101

6.8 Optical Readout Noise in Folded Interferometers . . . . . . . . . . 101

6.9 Transfer Function of a Folded Interferometer . . . . . . . . . . . 102

6.10 To Fold, or Not to Fold? . . . . . . . . . . . . . . . 105

$\begin{array}{ll}\text { Thermal Noise } & 107\end{array}$

7.1 Brownian Motion . . . . . . . . . . . . . 107

7.2 Brownian Motion of a Macroscopic Mass Suspended in a Dilute Gas . 108

7.3 The Fluctuation-Dissipation Theorem . . . . . . . . . . . 110

7.4 Remarks on the Fluctuation-Dissipation Theorem . . . . . . . . 112

7.5 The Quality Factor, $Q \ldots \ldots \ldots \ldots \ldots \ldots \ldots$

7.6 Thermal Noise in a Gas-Damped Pendulum . . . . . . . . . . 114

7.7 Dissipation from Internal Friction in Materials . . . . . . . . . 116

7.8 Special Features of the Pendulum . . . . . . . . . . . . . 121

7.9 Thermal Noise of the Pendulum's Internal Modes . . . . . . . . . 124 
8 Seismic Noise and Vibration Isolation $\quad 127$

8.1 Ambient Seismic Spectrum . . . . . . . . . . . . . . . . . . . 127

8.2 Seismometers . . . . . . . . . . . . . . . . . . 129

8.3 Vibration Isolators . . . . . . . . . . . . . . . 130

8.4 Myths About Vibration Isolation . . . . . . . . . . . . 132

8.5 Isolation in an Interferometer . . . . . . . . . . . . 133

8.6 Stacks and Multiple Pendulums . . . . . . . . . . . . . 136

8.7 Q: High or Low? . . . . . . . . . . . . . . . . . . . . . . 139

8.8 A Gravitational "Short Circuit" Around Vibration Isolators . . . . . 141

8.9 Beyond Passive Isolation . . . . . . . . . . . . . . . . 142

9 Design Features of Large Interferometers 145

9.1 How Small Can We Make a Gravitational Wave Inteferometer? . . . 145

9.2 Noise from Residual Gas . . . . . . . . . . . . . . . . 147

9.2 .1 Simple model . . . . . . . . . . . . . . . . . 149

9.2 .2 Exact result . . . . . . . . . . . . . . . . 150

9.2.3 Implications for Interferometer Design . . . . . . . . 150

9.3 The Space-Borne Alternative . . . . . . . . . . . . . . . 151

$\begin{array}{lc}10 \text { Null Instruments } & 153\end{array}$

10.1 Some virtues of nullity . . . . . . . . . . . . . . . 154

10.1.1 Null hypotheses . . . . . . . . . . . . . . . . 154

10.1 .2 Null experiments . . . . . . . . . . . . . . . 154

10.1.3 Null instruments . . . . . . . . . . . . . . 156

10.1.4 Null features of a gravitational wave interferometer . . . . 158

10.1.5 Active null instruments . . . . . . . . . . . . . 159

10.2 The Advantages of Chopping . . . . . . . . . . . . 162

10.3 The Necessity to Operate a Gravitational Wave Interferometer as an

Active Null Instrument . . . . . . . . . . . . . . . . . . . 164

10.3.1 The need to chop . . . . . . . . . . . . . . . 164

10.3.2 The need to actively null the output . . . . . . . 165

11 Feedback control systems 169

11.1 The Loop Transfer Function . . . . . . . . . . . . . . . . 171

11.2 The Closed Loop Transfer Function . . . . . . . . . . . . . . 172

11.3 Designing the Loop Transfer Function . . . . . . . . . . . . . 174

11.4 Instability . . . . . . . . . . . . . . . . 175

11.4.1 Causes of instability . . . . . . . . . . . 177

11.4.2 Stability tests . . . . . . . . . . . . . . . 179

11.5 The Compensation Filter . . . . . . . . . . . . . . . 179

11.6 Active Damping: A Servo Design Example . . . . . . . . 181

11.7 Feedback to Reduce Seismic Noise over a Broad Band . . . . . . . . 188

11.7.1 Suspension point interferometer . . . . . . . . . 188 
11.7.2 Active isolation . . . . . . . . . . . . . 189

12 An Interferometer as an Active Null Instrument 191

12.1 Fringe-Lock in a Non-Resonant Interferometer . . . . . . . . . . . . 191

12.2 Shot Noise in a Modulated Interferometer . . . . . . . . . . . 198

12.3 Rejection of Laser Output Power Noise . . . . . . . . . . . . . . . . 199

12.4 Locking the Fringe . . . . . . . . . . . . . . . . . . . . . . . 199

12.5 Fringe Lock for a Fabry-Perot Cavity . . . . . . . . . . . . . . . 203

12.6 A Simple Interferometer with Fabry-Perot Arms . . . . . . . . . 206

12.7 Beyond the Basic Interferometer . . . . . . . . . . . . . . . 208

12.7.1 Power recycling . . . . . . . . . . . . . . 208

12.7 .2 Signal recycling . . . . . . . . . . . . . . . . . . . 210

12.7.3 Resonant sideband extraction . . . . . . . . . . . 213

Resonant Mass Gravitational Wave Detectors 215

13.1 Does Form Follow Function? . . . . . . . . . . . . . . . . . . . . 215

13.2 The Idea of Resonant Mass Detectors . . . . . . . . . . . . . . 216

13.3 A Bar's Impulse Response and Transfer Function . . . . . . . . . . 217

13.4 Resonant Transducers . . . . . . . . . . . . . . . . . . . 222

13.5 Thermal Noise in a Bar . . . . . . . . . . . . . . . . . . . . . 224

13.6 Bandwidth of Resonant Mass Detectors . . . . . . . . . . . . . 228

13.6.1 When are narrow bandwidths optimum? . . . . . . . . 228

13.6.2 Interpreting narrow-band observations . . . . . . . 230

13.7 A Real Bar . . . . . . . . . . . . . . . . . . . . . . 231

13.8 Quantum Mechanical Sensitivity "Limit" . . . . . . . . . . . . . 232

13.9 Beyond the Quantum "Limit"? . . . . . . . . . . . . . . . 235

Detecting Gravitational Wave Signals $\quad \mathbf{2 3 7}$

14.1 The Signal Detection Problem . . . . . . . . . . . . . . 237

14.2 Probability Distribution of Time Series . . . . . . . . . 238

14.3 Coincidence Detection . . . . . . . . . . . . . . . 244

14.4 Optimum Orientation . . . . . . . . . . . . . . 245

14.5 Local Coincidences . . . . . . . . . . . . . . . . . . . . . . . . 246

14.6 Searching for Periodic Gravitational Waves . . . . . . . . . . . 247

14.6.1 When is a spectral peak improbably strong? . . . . . . . . . 247

14.6.2 Signatures of periodic gravitational waves . . . . . . . . . 247

14.6.3 Frequency noise in the source and elsewhere . . . . . . . . 251

14.7 Searching for a Stochastic Background . . . . . . . . . . 252

15 Gravitational Wave Astronomy 255

15.1 Gravitational Wave Source Positions . . . . . . . . . . . 255

15.1.1 Network figure of merit . . . . . . . . . . . . . 257

15.1.2 Why measure positions? . . . . . . . . . . . 259 
15.1.3 Inferences from precise positions . . . . . . . . . . . 261

15.1.4 Temporal coincidence with non-gravitational observations . . . 263

15.2 Interpretation of Gravitational Waveforms . . . . . . . . . . 264

15.2.1 Core collapse . . . . . . . . . . . . . . . . 265

15.2.2 Binary coalescences . . . . . . . . . . . . 265

15.2.3 A gravitational standard candle . . . . . . . . . 266

15.2.4 Recognizing signals from black holes . . . . . . . . 267

15.3 Previous Gravitational Wave Searches . . . . . . . . . . . . . . 268

15.3.1 Room temperature bars . . . . . . . . . . . 268

15.3.2 Cryogenic bars . . . . . . . . . . . . . . . . 269

15.3.3 The Strange case of Supernova 1987A . . . . . . . . . . . 271

15.3.4 Gravitational wave searches with interferometers . . . . . . . 273

15.3.5 Other observational upper limits . . . . . . . . . 274

$\begin{array}{ll}16 \text { Prospects } & \mathbf{2 7 7}\end{array}$

16.1 A Prototype Interferometer . . . . . . . . . . . . . 277

16.2 LIGO . . . . . . . . . . . . . . . . . . . . 279

16.3 Proposed Features of $4 \mathrm{~km}$ Interferometers . . . . . . . . . . 279

$\begin{array}{ll}\text { References } & 283\end{array}$

$\begin{array}{lr}\text { Index } & 295\end{array}$ 
This page is intentionally left blank 


\section{List of Figures}

2.1 An illustration of the two polarizations of a gravitational wave. . . 11

2.2 A thought-experiment version of a light clock. . . . . . . . . . 13

2.3 A schematic diagram of the simplest form of Michelson interferometer. 14

2.4 An impressionistic rendering of the appearance of interference fringes at the output of a classic Michelson interferometer. . . . . . . . . . 17

2.5 The transfer function of a simple Michelson interferometer. . . . . . . 22

2.6 Coordinates used to describe the antenna pattern of a gravitational wave detector. . . . . . . . . . . . . . . . . . . 23

2.7 The sensitivity, as a function of direction, of an interferometric gravitational wave detector to unpolarized gravitational waves. . . . . . . 24

2.8 A thought-experiment version of a resonant mass detector of gravitational waves, or "bar". ................. 26

3.1 A schematic representation of a binary star system. . . . . . . . 31

3.2 A schematic diagram of a rotating dumbbell, a thought-experiment version of a laboratory generator of gravitational waves. . . . . . . . 35

4.1 The cross-correlation operation. . . . . . . . . . . . . . 51

4.2 A functional block diagram of a filter-based spectrum analyzer. . . $\quad 54$

4.3 A schematic diagram of a mechanical oscillator. . . . . . . . . 56

4.4 The impulse response of our mechanical oscillator. . . . . . . . . 58

4.5 The convolution operation. . . . . . . . . . . . . 59

4.6 A Bode plot of the transfer function of the mechanical oscillator. . . 63

4.7 Two versions of the histogram of a variable with a Gaussian probability distribution. ...................... 65

5.1 The output power from a Michelson interferometer, as a function of the difference in arm lengths. . . . . . . . . . . . . 73

5.2 A thought-experiment version of the Heisenberg microscope. . . . . 76

5.3 The optical readout noise of a Michelson interferometer. . . . . . . 79

6.1 A schematic diagram of an optical waveguide constructed from lenses. 86

6.2 A schematic diagram of a Herriott delay line. . . . . . . . . . . . 87

6.3 A schematic diagram of a Fabry-Perot cavity. . . . . . . . . . . 92 
6.4 The power transmitted by a Fabry-Perot cavity in the vicinity of a resonance, as a function of the offset from resonance $\mathrm{kL}$. . . . . . . .

6.5 The phase of the light reflected from a Fabry-Perot cavity in the vicinity of a resonance, as a function of the offset from resonance kL. . . . . . 94

6.6 A phasor diagram of the light reflected from a Fabry-Perot cavity. . . 96

6.7 A phasor diagram illustrating the effect of a small amount of scattered light interfering with the main beam in an interferometer. . . . . . . .

6.8 Transfer functions of interferometric gravitational wave detectors made with delay lines and with Fabry-Perot cavities for arms. . . . . . . . 104

7.1 A schematic diagram of a macroscopic mass suspended in a dilute gas. 109

7.2 Three different manifestations of the quality factor $\mathrm{Q}$ of an oscillator. 115

7.3 The strain noise spectrum of thermal noise due to residual gas damping.117

7.4 A schematic representation of a Maxwell unit, the basic building block of the description of anelasticity. . . . . . . . . . . . .

7.5 The real and imaginary parts of the the spring constant of a spring damped by a single relaxation.

7.6 The strain noise spectral density due to thermal noise from internal friction in the pendulum wires.

7.7 The strain noise spectral density due to thermal noise of the internal normal modes of the interferometer test masses.

8.1 The displacement spectral density of the ground at a relatively quiet site.

8.2 The magnitude of the transfer function of a vibration isolator whose resonant frequency is $1 \mathrm{~Hz}$.

8.3 The strain noise spectral density due to seismic noise in a $4 \mathrm{~km}$ interferometer, if the only vibration isolation were that provided by $1 \mathrm{~Hz}$ pendulum suspensions of the test masses.

8.4 The transfer function of a pendulum, when the effect of resonances in the wires is included in the model.

8.5 A schematic diagram of a vibration isolation stack, constructed of metal plates separated by springs.

8.6 An estimate of the strain noise spectral density in a $4 \mathrm{~km}$ interferometer, when the pendulum isolation is supplemented by a multi-layer stack of the sort described in the text.

8.7 The strain noise spectral density in a $4 \mathrm{~km}$ interferometer due to local gravitational noise.

9.1 The strain noise spectral density in inteferometers with test mass separations $\mathrm{L}$ of $40 \mathrm{~m}, 400 \mathrm{~m}$, and $4 \mathrm{~km}$, respectively. . . . . . . . 
10.1 Two circuits for determining the value of an unknown resistance: a) a voltage divider, and b) a bridge.

10.2 A schematic diagram of a Dicke radiometer, an example of a null instrument.

10.3 An example of a noise spectrum exhibiting white noise at high frequencies, with a component of $1 / \mathrm{f}$ noise at low frequencies.

10.4 A block diagram showing the parts of a generic feedback control system. 166

11.1 Each component of a feedback control system can be characterized by its transfer function.

11.2 One method of measuring a transfer function, by comparing a system's output to its input using a dual-trace oscilloscope.

11.3 The signals necessary for understanding the closed loop transfer function of a feedback control system.

11.4 A palette of impulse responses of a linear system. . . . . . . . 176

11.5 A Bode plot of the transfer function of a block of elastic solid. . . . 178

11.6 A schematic diagram of a feedback system to damp the response of a

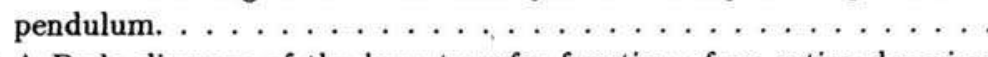

11.7 A Bode diagram of the loop transfer function of an active damping system. . . . . . . . . . . . . . . .

11.8 The open loop and closed loop seismic transfer functions of a pendulum fitted with an active damping system. . . . . . . . . .

11.9 A block diagram of a generic feedback control system, showing how sensor noise enters.

11.10A schematic diagram of a passive vibration isolation system that has been equipped with a feedback system for active isolation.

12.1 A schematic diagram of a Michelson interferometer equipped with Pockels cell phase modulators in each arm. . . . . . . . . . .

12.2 The output power of an interferometer as a function of path length difference, showing the locations of the "naive" operating point and the dark fringe.

12.3 The Bessel functions of order 0 through $3 . \ldots \ldots 196$

12.4 An illustration of how laser power fluctuations would cause interferometer output noise, if the system does not sit at the dark fringe. .

12.5 A schematic diagram of a Michelson interferometer equipped with a fringe-locking servo. . . . . . . . . . . . . . .

12.6 A schematic diagram of the components used in the reflection cavity locking scheme.

12.7 A phasor diagram illustrating the addition of the carrier field and the modulation field: a) with the light's wavelength centered on the cavity resonance, and b) with the wavelength offset from resonance. 
12.8 A schematic diagram of the "simple" interferometer made with two Fabry-Perot cavities.

12.9 A schematic diagram of a Michelson interferometer in which the light is recycled.

12.10A schematic diagram of a Michelson interferometer that uses signal recycling in addition to light recycling.

13.1 A schematic diagram of a model of a resonant mass gravitational wave detector. . . . . . . . . . . . . . . . . . 218

13.2 Two views of the impulse response of a bar to an external force. . . . 219

13.3 A Gaussian impulse in $\mathrm{h}$, and the corresponding force across a bar. . 220

13.4 The transfer function of a bar to an external force, shown in the vicinity of the resonance.

13.5 A schematic diagram of a model of a bar with a resonant transducer attached to one end. . . . . . . . . . . . . . . . . . . 223

13.6 The total noise spectrum of a bar in the vicinity of the resonance. . . 226

13.7 Matched templates for the two quadratures of a bar's oscillation. . . . 227

13.8 Schematic diagrams of the noise sources in a) a current amplifier and b) a voltage amplifier.

14.1 The complementary error function. . . . . . . . . . . . . . . 241

14.2 The histogram of a variable with Gaussian noise. . . . . . . . . . 243

15.1 A diagram of the celestial sphere (seen from outside!), on which is drawn the position circle from the difference in the arrival times of a signal at two gravitational wave detectors.

15.2 Detection of a signal by three instruments gives two time differences.

15.3 The locations of three gravitational wave detectors on the terrestrial globe, illustrating the area figure of merit for a network. . . . . . .

15.4 The volume figure of merit for a network of four gravitational wave detectors.

15.5 Upper limits set by various experiments on the rate of gravitational wave impulse arrivals, as a function of the characteristic amplitude of the impulse.

16.1 Recent noise spectrum of the 40 meter interferometer at Caltech. . . . 278

16.2 An estimate of the strain noise spectral density in an early LIGO interferometer. 


\section{Preface}

This book is an attempt to communicate the basic logic of interferometric gravitational wave detectors to people who are new to the field. With the recent start of construction of the Laser Interferometer Gravitational Wave Observatory in the United States, and similar ventures elsewhere, it seems likely that many people will want to know more about the subject, including both young people at the beginnings of their scientific careers and experienced scientists with a new interest in an expanding field. While I in no way expect that this book will displace the more specialized literature, I hope it will serve as an overview of a subject of tremendous intellectual excitement and technical sweetness. I assume the reader has a basic knowledge of physics, but no special familiarity with either general relativity or the special techniques of experimental physics.

\section{... a grateful mind}

By owing owes not, but still pays, at once

Indebted and discharged; what burden then?

Paradise Lost, IV:55-7

The inspiration for this book came from Abhay Ashtekar. As he promised, the Physics Department at Syracuse University has proven to be a wonderful home at which to teach, to learn, and to write. The outline of this book was worked out during a seminar I conducted there with my research group. Large parts of the manuscript were read carefully by Stan Whitcomb and by Gabriela Gonzalez; crucial parts were also read by John Price, David Shoemaker, and Robert Spero. Yinglei Huang, Alex Evako, Joe Adams, and especially Gabriela Gonzalez were of tremendous help in improving the presentation, tracking down important facts and ferreting out mistakes. Bess Collins taught me how to look at the figures from the point of view of a graphic designer. Everyone named above made valuable suggestions for which I am grateful. I am of course responsible for all of the mistakes that remain.

I was privileged to be able to present the contents of Chapters 2 and 5 of the present volume as lectures in the School of Cosmology and Gravitation at Erice in May, 1993. My thanks go to Joseph Weber for the invitation to lecture there, and to the School's organizer Venzo de Sabbata and the staff at Erice for creating such a pleasant ambiance for the study of physics. 
It is with profound gratitude that I thank here those who have helped to shape me as a physicist. I was inspired to study physics by Edward Purcell, and guided along the way by Paul Bamberg, David Layzer, and Herbert Gursky. My graduate apprenticeship was in the hands of David Wilkinson, a model of what a scientist should be. In the Gravity Group at Princeton, the inspiring example of Robert Dicke $\Xi \frac{0}{S}$ was never far away. I learned the trade of gravitational wave detection from Rainer Weiss; Rai not only initiated me into the mysteries of the field, but also set standards of intellectual rigor and personal responsibility that are a continuing model.

I have also learned a great deal from the graduate students alongside of whom I have been privileged to work; at MIT, this included Dan Dewey, Jeff Livas, David Shoemaker, Michelle Stephens, Joe Kovalik, Nelson Christensen, Peter Fritschel, and Joe Giaime. Throughout my career, it has been my privilege to be surrounded by colond leagues and students who were a constant source of insight and inspiration; the talent 政 Ji Thanks to you all.

This work was supported in part by the National Science Foundation, through grant PHY-9113902. I am grateful to the American taxpayers for underwriting this of field of research, and for the responsible care exercised on their behalf by Richard 章Isaacson.

For support in all possible forms over many years, I thank my parents, Dr. Stanley H. Saulson and Helen M. Saulson. I owe my deepest thanks to my wife, Sarah F. Saulson, for her love, encouragement, and fine editorial ear. 